\title{
UTILIZAÇÃO DE RESÍDUOS DE MADEIRA DE Pinus spp. CINZA VOLANTE E ESCÓRIA DE ALTO-FORNO EM COMPÓSITOS CIMENTO-MADEIRA
}

\author{
Adauto José Miranda de Lima ${ }^{1}$, Setsuo Iwakiri ${ }^{2}$ \\ ${ }^{1}$ Eng. Civil, Dr., Depto. de Construção Civil, UTFPR, Curitiba, PR, Brasil - adautojm@ @utfpr.edu.br \\ ${ }^{2}$ Eng. Florestal, Dr., Depto. de Engenharia e Tecnologia Florestal, UFPR, Curitiba, PR, Brasil - setsuo@ufpr.br \\ Recebido para publicação: 15/04/2010 - Aceito para publicação: 02/06/2011
}

\begin{abstract}
Resumo
Este trabalho teve como objetivo avaliar o potencial de utilização do resíduo do desdobro de toras de Pinus spp. e de substituições parciais do cimento Portland por cinza volante e escória de alto-forno, em compósitos cimento-madeira. As características físicas (massa específica, índice de vazios e absorção de água) e mecânicas (resistência à compressão e à tração) dos compósitos foram determinadas conforme normas da ABNT. Foram avaliadas substituições do cimento Portland por teores de $5 \%$ a $40 \%$ de cinza volante e $25 \%$ a $60 \%$ de escória de alto-forno. Em ambos os casos, foi utilizado aditivo químico para acelerar a pega do cimento $\left(\mathrm{CaCl}_{2} \cdot 2 \mathrm{H}_{2} \mathrm{O}\right)$. Os resultados demonstraram viabilidade técnica da utilização do resíduo de Pinus spp. e das substituições do cimento Portland do compósito, sendo que os valores otimizados, determinados através de análise multivariada, foram de $5 \%$ de cinza volante e $25 \%$ de escória de alto-forno.
\end{abstract}

Palavras-chave: Compósito cimento-madeira; Pinus spp; cinza volante; escória de alto-forno.

\begin{abstract}
Uses of wood residues of Pinus spp. fly ash and blast slag furnace in cement-wood composites. This research aimed to evaluate the potencial use of residues of Pinus spp. sawnwood production and partial substitutions of Portland cement for fly ash and blast slag furnace, in cement-wood composites. The physical characteristics (density, porosity and water absorption) and mechanical characteristics (compression and traction resistances) of the composites were evaluated in agreement with the ABNT normalization. The amount of substituition to the Portland cement for $5 \%$ to $40 \%$ of fly ash and $25 \%$ to $60 \%$ of blast slag furnace were evaluated. In both cases chemical addictive, to accelerate de cement hydration $\left(\mathrm{CaCl}_{2} \cdot 2 \mathrm{H}_{2} \mathrm{O}\right)$, were used. The results demonstrated the technical feasibility to residue of Pinus spp. use, and substitutions of Portland cement in composite, accordingly to optimized values obtained by multivariate statictical analysis, were $5 \%$ of fly ash and $25 \%$ of blast slat furnace.
\end{abstract}

Keywords: Cement-wood composites; Pinus spp.; fly ash; blast slag furnace.

\section{INTRODUÇÃO}

A viabilidade da produção de compósitos produzidos com fibras e/ou partículas naturais vem sendo pesquisada há décadas, com longa história de aceitação e aplicação na construção civil, especialmente na Europa e Ásia.

De acordo com Semple e Evans (2004) e Moslemi (1989), os materiais produzidos com compósitos cimento-madeira apresentam uma série de vantagens, entre as quais pode ser citada a disponibilidade de matéria-prima em grandes quantidades e baixa massa específica, o que permite sua utilização na produção de painéis de fechamento, forros, telhas, elementos de vedação e de alvenaria. Além desses atributos, os materiais podem ser serrados, furados e receber parafusos e pinturas.

Somando-se a essas vantagens, os materiais à base de compósitos cimento-madeira não emitem resíduos tóxicos durante sua manufatura (VAN ELTEN, 2000 apud SEMPLE; EVANS, 2004), não necessitam de tratamentos preservativos (RAMIREZ-CORETTI et al., 1998) e apresentam menor dispêndio de energia no processo de produção (MOSLEMI, 1989), tornando-se uma alternativa viável aos 
fibrocimentos que utilizam asbesto (amianto), principalmente na Europa, Japão e América do Norte (MOSLEMI, 1989).

O gênero Pinus spp. da família das Pinaceae, é composto por plantas lenhosas, em geral arbóreas, de altura variável, sendo que algumas espécies de Pinus se incluem entre as árvores mais utilizadas no mundo (LIMA et al., 1988; MORAIS et al., 2005).

As madeiras pertencentes ao gênero Pinus spp. fornecem material de boa qualidade para a produção de celulose, além de apresentarem características tecnológicas adequadas para seu aproveitamento em serraria. Durante as etapas do processamento mecânico da madeira, produz-se grande quantidade de resíduos, que, de acordo com Nahuz (2004), até o momento não têm recebido a devida valorização.

O incremento na produção de madeira de Pinus spp. pode ser verificado com base na análise dos dados da Associação Brasileira da Indústria de Madeira Processada Mecanicamente (ABIMCI, 2009), pelos quais se verifica uma produção de toras de Pinus spp. em 2007 de 50,6 milhões de metros cúbicos, contra 42 milhões de metros cúbicos em 1997. Portanto, um acréscimo de produção de 20,48\% no período de dez anos.

Sob essa ótica, a geração de resíduos florestais no Brasil também assume valores expressivos, pois, segundo a ABIMCI (2009), só a produção de madeira serrada em 2008 alcançou o montante de 22,886 milhões de metros cúbicos. Considerando o potencial de perdas, que, conforme Freitas (2000), pode atingir até $60 \%$, deduz-se que um valor significativo de resíduos florestais foi produzido naquele ano.

Dentre os resíduos de outras atividades industriais, alguns, como a cinza volante e a escória de alto-forno, apresentam propriedades que permitem o uso como substituintes ao cimento Portland, cuja produção, conforme Oliveira (2000), é responsável por 7\% das emissões de $\mathrm{CO}_{2}$ no planeta. A utilização desses dois materiais cimentícios alternativos já é prática consolidada na produção do cimento Portland, em que são utilizados como materiais cimentícios complementares, adicionados sob a forma de pó, após a produção do clínquer.

A capacidade dos materiais cimentícios alternativos em reagir com o $\mathrm{Ca}(\mathrm{OH})_{2}$, também conhecida como atividade pozolânica, é propriedade fundamental para a utilização desses materiais (SANTOS, 1992). Essa reação resulta na formação de compostos estáveis de poder aglomerante, tais como os silicatos e os aluminatos de cálcio hidratado, que são os principais elementos responsáveis pela resistência das pastas hidratadas de cimento (CARMO, 2006). Essa reação, por se dar a temperatura ambiente, é geralmente lenta e pode levar meses para ser completada, entretanto, quanto mais vítrea e mais fina for a pozolana, mais rápida será a reação com o $\mathrm{Ca}(\mathrm{OH})_{2}$ do cimento.

John e Agopyan (1993) afirmam que o emprego de materiais cimentícios alternativos pode contribuir para um aumento da durabilidade de compósitos com fibras ou partículas vegetais. Com o consumo de parte do $\mathrm{Ca}(\mathrm{OH})_{2}$ que está concentrado na zona de transição, ocorre a melhora da aderência entre fibra ou partícula vegetal e matriz, além do aumento da durabilidade do compósito, que, conforme Lange et al. (1989), se deve à redução do pH da matriz cimentícia, em função da combinação dos íons $\mathrm{Ca}^{2+}$ liberados durante a hidratação do cimento.

As cinzas volantes são o resíduo da queima de carvão mineral em pó, proveniente de fornos que usam carvão mineral moído como combustível. O pó, bastante nocivo ao meio ambiente, é coletado por filtros de mangas ou filtros eletrostáticos. A produção mundial de cinzas volantes é estimada em mais de 700 milhões de toneladas/ano, das quais $70 \%$ (cerca de 500 milhões de toneladas) são adequadas e podem ser utilizadas como adição em concretos e outros produtos de cimento (FREITAS JÚNIOR, 2005).

A utilização de cinzas volantes pode resultar em um retardamento da velocidade do ganho de resistência mecânica nas primeiras idades dos compósitos cimentícios (até os 28 dias), porém, posteriormente, a resistência alcançada se iguala e até ultrapassa a do clínquer hidratado (MEHTA; MONTEIRO, 2008).

No Brasil, a maior fração do uso dessas adições é na composição dos cimentos Portland tipos CP II Z - cimento Portland composto com adição de pozolana, conforme norma NBR 11.578 (1991), e CP IV - cimento Portland pozolânico, conforme a norma NBR 5.736 (1991).

A escória de alto-forno é o subproduto da manufatura do ferro gusa num alto-forno. Todas as impurezas contidas no minério de ferro e no coque passam para a escória de alto-forno. Como todas essas impurezas poderiam resultar numa mistura com ponto de fusão muito alto, agentes fundentes são adicionados à carga do alto-forno, de modo que a composição química resultante das impurezas fique 
dentro de uma região muito bem definida do diagrama de fases $\mathrm{SiO}_{2}-\mathrm{CaO}-\mathrm{Al}_{2} \mathrm{O}_{3}$, correspondendo a uma das duas áreas de temperatura de fusão mais baixas dentro desse diagrama. Assim, do ponto de vista químico, a escória tem uma composição muito constante, pois qualquer alteração traduz-se em demandas significativas de energia e custos finais (AÏTCIN, 2000).

O ferro gusa é a matéria-prima para a produção do aço e, conforme Isaia (2005), a produção brasileira em 2005 foi de cerca de 35 milhões de toneladas. Conforme Oliveira (2000), dessas operações, para a produção de uma tonelada de ferro gusa resulta a obtenção de cerca de $300 \mathrm{~kg}$ de escória granulada de alto-forno, o que pode representar, com base nesses dados, a produção de 10,5 milhões de toneladas de escória de alto-forno para aquele ano. Atualmente, a maior parte da escória de alto-forno brasileira é usada como matéria-prima na indústria cimenteira, para a produção dos cimentos Portland tipo CP II E cimento Portland composto com adição de escória, conforme a NBR 11.578 (1991), e CP III - cimento Portland de alto-forno, conforme a NBR 5735 (1991).

Os aceleradores de pega são compostos orgânicos ou inorgânicos solúveis em água utilizados para propiciar uma redução do tempo de cura e aumento da velocidade de desenvolvimento da resistência inicial do material (MEHTA; MONTEIRO, 2008).

Conforme Isaia (2005), entre os compostos inorgânicos encontram-se os sais de elementos alcalinos ou alcalino-terrosos à base de cloretos, fluoretos, carbonatos, silicatos, fluorsilicatos, aluminatos, boratos, nitratos, nitritos, tiossulfatos etc. Para Mehta e Monteiro (2008), o aditivo acelerador de pega mais conhecido e amplamente utilizado é o cloreto de cálcio $\left(\mathrm{CaCl}_{2}\right.$.).

Tendo em vista a importância de usos alternativos de resíduos de madeira e outros materiais minerais em produtos de maior valor agregado, no presente trabalho avaliou-se a hipótese da possibilidade de substituições parciais do cimento Portland por cinza volante e escória de alto-forno na produção de compósitos cimento-madeira.

\section{MATERIAIS E MÉTODOS}

O resíduo de madeira de Pinus spp. utilizado neste estudo foi proveniente de uma madeireira localizada no município de Almirante Tamandaré (PR). Após a coleta, o resíduo foi seco ao ar e peneirado em uma peneira $\mathrm{ABNT} \mathrm{n}^{\circ}$ 4, de forma a garantir ao resíduo de Pinus spp. uma granulometria contínua, com dimensão máxima característica (Dmáx) de 4,8 mm, similar à do agregado miúdo mineral (areia).

Na produção dos compósitos de referência, o resíduo de Pinus spp. foi utilizado in natura, ou seja, sem qualquer pré-tratamento ou uso de aditivos químicos (Referência 1), in natura e com 4,5\% de $\mathrm{CaCl}_{2} \cdot 2 \mathrm{H}_{2} \mathrm{O}$ (Referência 2 ) e com pré-tratamento tipo extração em água fria por 48 horas $(\mathrm{AF}-48 \mathrm{H})$ e com $4,5 \%$ de $\mathrm{CaCl}_{2} \cdot 2 \mathrm{H}_{2} \mathrm{O}$ (Referência 3 ).

O aglomerante utilizado na pesquisa foi o cimento Portland tipo CPV ARI RS - de Alta Resistência Inicial - Resistente a Sulfatos, especificado pela norma ABNT NBR 5.733 (1991).

A cinza volante utilizada foi oriunda da queima do carvão mineral da Usina Termelétrica de Charqueadas, Charqueadas (RS). A escória de alto-forno utilizada foi oriunda da Companhia Siderúrgica Paulista - COSIPA, Cubatão (SP).

Os materiais cimentícios alternativos submetidos à fluorescência de raios-x apresentaram teores de $\mathrm{SiO}_{2}+\mathrm{Al}_{2} \mathrm{O}_{3}+\mathrm{Fe}_{2} \mathrm{O}_{3}$ acima do limite de 50\% imposto pela NBR 12.653 (1992). Os teores de $\mathrm{SO}_{3}$ e $\mathrm{Na}_{2} \mathrm{O}_{3}$, por sua vez, se apresentaram abaixo dos limites impostos pela norma brasileira.

$\mathrm{O}$ aditivo acelerador de pega utilizado foi o cloreto de cálcio bi-hidratado, $\mathrm{CaCl}_{2} \cdot 2 \mathrm{H}_{2} \mathrm{O}$.

A confecção dos corpos de prova cilíndricos, com diâmetro de $50 \mathrm{~mm}$, baseou-se na Norma Brasileira NBR 7215 (1996). Foi utilizado o traço apresentado por Iwakiri (2005), fixando-se uma relação madeira/cimento de 13,3:1 e uma relação água/cimento de 0,35 . O teor de água da mistura foi controlado e corrigido em função do teor de umidade do resíduo de Pinus spp., de forma a manter sempre a madeira no ponto de saturação das fibras (PSF), adotado neste estudo como $30 \%$.

A amostra mínima a ser ensaiada, a fim de garantir estatisticamente a distribuição e confiabilidade dos resultados, foi determinada pela equação:

$$
n=z_{\alpha / 2}^{2} \cdot \frac{C V^{2}}{E r^{2}}
$$


Em que: $\mathrm{n}=$ número de repetições;

$\mathrm{Er}=$ erro relativo admitido da estimativa, fixado em $10 \%$;

$\mathrm{CV}=$ coeficiente de variação da amostra;

$\mathrm{z}_{\alpha / 2}=$ valor tabelado que, para um nível $\alpha=5 \%$ de significância, possui o valor de 1,96.

A cura inicial dos corpos de prova, com duração de 24 horas, deu-se em câmara úmida, conforme NBR 9.479 (2006), com temperatura de $23 \pm 2{ }^{\circ} \mathrm{C}$ e umidade relativa mínima de $95 \%$, onde permaneceram com os moldes e cobertos com uma placa de acrílico, para proteção, conforme NBR 7.215 (1996). Após o período de cura inicial, os corpos de prova foram desmoldados, identificados e mantidos em câmara úmida, imersos em água, até a data de ensaio.

As características mecânicas, compreendendo os ensaios para determinação da resistência à compressão aos 7 e 91 dias, foram determinadas conforme NBR 7.215 (1997). Para não haver influências de irregularidades nos topos dos corpos de prova e se garantir a ruptura por compressão simples, foram utilizados discos de neoprene com dureza 60 a 70 shore, dispostos em guarnições de aço. As características mecânicas dos compósitos produzidos nesta pesquisa foram determinadas em máquina universal de ensaio marca Emic, modelo DL 30000, com tomada de dados por computador. Os ensaios foram realizados no Labmat - Laboratório de Materiais de Construção, da Universidade Tecnológica Federal do Paraná (UTFPR).

Os teores de substituições parciais do cimento Portland dos compósitos cimento-madeira (resíduos de Pinus spp.) foram tomados com base na massa de cimento do traço e variaram em função do tipo da substituição, sendo 5\%, 10\%, 15\%, 20\%, 30\% e 40\% de cinza volante e $25 \%, 30 \%, 35 \%, 40 \%$, $50 \%$ e $60 \%$ de escória de alto-forno.

Quanto ao aditivo acelerador de pega, para cada teor de substituição do cimento Portland, foram testados teores de $\mathrm{CaCl}_{2} \cdot 2 \mathrm{H}_{2} \mathrm{O}$, variando de $0 \%, 1 \%, 2 \%, 3 \%$ e 4,5\%. $\mathrm{O}$ teor limite de 4,5\% foi adotado por ter apresentado o melhor desempenho nos compósitos com o resíduo de Pinus spp. in natura.

Com base nos resultados de resistência à compressão na idade de 7 dias (RC 7d) e 91 dias (RC 91d) dos compósitos produzidos com as substituições parciais do cimento Portland, efetuaram-se análises multivariadas através de regressões múltiplas, considerando-se como variáveis dependentes as resistências dos compósitos aos 91 dias de idade e como variáveis independentes os teores de substituição ao cimento Portland e os teores de $\mathrm{CaCl}_{2} \cdot 2 \mathrm{H}_{2} \mathrm{O}$. Essas composições otimizadas foram produzidas e testadas em laboratório, para determinação das características físicas e mecânicas dos compósitos.

As características físicas dos compósitos otimizados aos 28 dias de idade, compreendendo os ensaios para determinação da massa específica, índice de vazios e absorção de água, foram avaliadas conforme a norma NBR 9.778 (2005). As características mecânicas, compreendendo os ensaios para determinação da resistência à compressão aos 7 dias (RC 7d), 28 dias (RC 28d) e 91 dias (RC 91d), foram avaliadas conforme a NBR 7.215 (1997). A resistência à tração por compressão diametral aos 28 dias de idade (RT 28d) foi determinada conforme a NBR 7.222 (1994).

A análise estatística dos resultados obtidos nos ensaios foi feita através da análise de variância (ANOVA) e, todas as vezes que a hipótese da nulidade foi rejeitada, efetuou-se a comparação das médias através do teste de Tukey-Kramer.

\section{RESULTADOS E DISCUSSÃO}

\section{Análise da substituição parcial do cimento Portland pela cinza volante}

Os resultados dos ensaios de resistência à compressão, obtidos para 7 e 91 dias de idade dos compósitos com substituição parcial do cimento Portland pela cinza volante, estão apresentados na tabela 1.

Os maiores valores de RC 7d, para os compósitos produzidos com substituição parcial do cimento Portland, foram verificados nos compósitos para o teor de substituição com cinza volante $(\mathrm{CV})$ de $10 \%$ com adição de $1 \%, 2 \%$ e $3 \%$ de $\mathrm{CaCl}_{2} \cdot 2 \mathrm{H}_{2} \mathrm{O}$ e com $\mathrm{CV}$ de $15 \%$ com adição de $2 \%$ e $3 \%$ de $\mathrm{CaCl}_{2} \cdot 2 \mathrm{H}_{2} \mathrm{O}$. A análise estatística não indicou diferenças significativas entre as médias obtidas. Observase também que esses valores de RC 7d não diferiram estatisticamente em relação ao compósito Ref. 3, sendo, porém, significativamente superiores aos valores determinados para as Ref. 1 e 2 .

Como pode ser observado na tabela 1, os resultados da RC 91d dos compósitos produzidos com 5\% de CV foram significativamente superiores às resistências verificadas para os compósitos de Referência, produzidos com $100 \%$ de cimento Portland, e superiores, também, às resistências determinadas com os compósitos produzidos com outros teores de substituição do cimento por cinza volante (CV). 
Através de técnicas de análise multivariada, determinou-se o seguinte modelo para expressar a dependência da RC 91d em função dos teores de cinza volante e $\mathrm{CaCl}_{2} \cdot 2 \mathrm{H}_{2} \mathrm{O}$ :

$$
R C 91 d(M P a)=\left(-23273+221099 / L n([\text { Teor Cinza Volante }(\%)])-2,4348 \times 10^{-34} /[\text { Teor CaCl2 }(\%)]^{1 / 3}\right)^{1 / 3}
$$

O modelo apresentou fortíssima correlação $\left(R=0,9192, R^{2}=0,8450\right.$ e $\left.R_{\text {Ajust }}^{2}=0,8424\right)$, o que indica que os dados experimentais estão bem ajustados pela superfície de resposta. A confiabilidade do modelo, calculada com base na sua significância $\left(4,3 \times 10^{-46}\right)$, foi superior a $99,99 \%$.

Na figura 1 está apresentada a superfície de resposta da RC 91d do compósito com substituição parcial do cimento Portland CPV ARI RS em função do teor de substituição por cinza volante e do teor de $\mathrm{CaCl}_{2} \cdot 2 \mathrm{H}_{2} \mathrm{O}$, conforme modelo determinado pela análise multivariada.

Tabela 1. Variação da resistência à compressão aos 7 e 91 dias de idade em função da substituição parcial do cimento Portland por cinza volante e do teor de $\mathrm{CaCl}_{2} \cdot 2 \mathrm{H}_{2} \mathrm{O}$.

Table 1. Variation of compression strength to ages of 7 and 91 days in function of partial substitution of Portland cement by fly ash and amount of $\mathrm{CaCl}_{2} \cdot 2 \mathrm{H}_{2} \mathrm{O}$.

\begin{tabular}{|c|c|c|c|c|c|c|c|}
\hline \multirow[b]{2}{*}{ TS-CV } & \multirow{2}{*}{$\begin{array}{c}\text { T-CC } \\
(\%)\end{array}$} & \multirow{2}{*}{$\begin{array}{c}\text { T-SP } \\
(\%)\end{array}$} & \multirow{2}{*}{$\begin{array}{c}\text { CONS } \\
(\mathrm{mm})\end{array}$} & \multicolumn{4}{|c|}{ Resistência à compressão (MPa) } \\
\hline & & & & RC-7d & $\begin{array}{c}\text { CVar } \\
(\%)\end{array}$ & RC-91d & $C \operatorname{Var}(\%)$ \\
\hline $\mathrm{PN}^{1}$ & 0,00 & 0,00 & 283,00 & $20,01 \mathrm{Q}$ & 4,99 & $28,97 \mathrm{KL}$ & 5,68 \\
\hline $\mathrm{PNCC}^{2}$ & 4,50 & 0,00 & 257,00 & $32,57 \mathrm{FGH}$ & 1,90 & $39,82 \mathrm{CDE}$ & 5,46 \\
\hline PNAFCC $^{3}$ & 4,50 & 0,00 & 248,00 & $34,73 \mathrm{ABCD}$ & 2,48 & $43,91 \mathrm{~B}$ & 1,21 \\
\hline \multirow{5}{*}{5,0} & 0,00 & 0,00 & 255,00 & $29,57 \mathrm{IJK}$ & 5,93 & $42,07 \mathrm{BC}$ & 2,30 \\
\hline & 1,00 & 0,00 & 257,00 & $31,11 \mathrm{HI}$ & 7,46 & $48,25 \mathrm{~A}$ & 2,10 \\
\hline & 2,00 & 0,00 & 256,00 & $34,00 \mathrm{BCDEF}$ & 5,14 & $50,16 \mathrm{~A}$ & 1,93 \\
\hline & 3,00 & 0,00 & 258,00 & $32,78 \mathrm{EFGH}$ & 4,60 & $49,65 \mathrm{~A}$ & 2,87 \\
\hline & 4,50 & 0,00 & 244,00 & $31,78 \mathrm{GH}$ & 3,18 & $49,35 \mathrm{~A}$ & 2,35 \\
\hline \multirow{5}{*}{10,0} & 0,00 & 0,00 & 275,00 & $32,23 \mathrm{FGH}$ & 1,73 & $32,03 \mathrm{IJ}$ & 5,31 \\
\hline & 1,00 & 0,00 & 259,00 & $34,63 \mathrm{ABCD}$ & 3,26 & $37,71 \mathrm{EF}$ & 1,75 \\
\hline & 2,00 & 0,00 & 257,00 & $35,69 \mathrm{AB}$ & 1,88 & $37,69 \mathrm{EF}$ & 3,47 \\
\hline & 3,00 & 0,00 & 265,00 & $34,94 \mathrm{ABC}$ & 2,03 & 43,42 B & 3,24 \\
\hline & 4,50 & 0,00 & 248,00 & $33,71 \mathrm{CDEF}$ & 1,95 & $40,45 \mathrm{CD}$ & 4,66 \\
\hline \multirow{5}{*}{15,0} & 0,00 & 0,00 & 255,00 & $27,81 \mathrm{KL}$ & 3,34 & $35,76 \mathrm{FG}$ & 3,99 \\
\hline & 1,00 & 0,00 & 263,00 & $33,03 \mathrm{DEFG}$ & 2,75 & $38,73 \mathrm{DE}$ & 7,08 \\
\hline & 2,00 & 0,00 & 265,00 & $36,09 \mathrm{~A}$ & 3,51 & $40,86 \mathrm{CD}$ & 5,51 \\
\hline & 3,00 & 0,00 & 255,00 & $34,40 \mathrm{ABCDE}$ & 3,53 & $39,26 \mathrm{DE}$ & 3,52 \\
\hline & 4,50 & 0,00 & 247,00 & $31,76 \mathrm{GH}$ & 5,61 & $39,67 \mathrm{DE}$ & 7,02 \\
\hline \multirow{5}{*}{20,0} & 0,00 & 0,00 & 265,00 & $28,05 \mathrm{JKL}$ & 4,78 & 32,68 HIJ & 4,90 \\
\hline & 1,00 & 0,00 & 272,00 & $33,25 \mathrm{CDEFG}$ & 4,41 & $40,45 \mathrm{CD}$ & 2,21 \\
\hline & 2,00 & 0,00 & 275,00 & $33,59 \mathrm{CDEF}$ & 1,29 & $39,08 \mathrm{DE}$ & 5,30 \\
\hline & 3,00 & 0,00 & 270,00 & $29,81 \mathrm{IJ}$ & 4,78 & $37,94 \mathrm{EF}$ & 6,85 \\
\hline & 4,50 & 0,00 & 253,00 & $28,11 \mathrm{JKL}$ & 5,14 & $34,85 \mathrm{GH}$ & 6,09 \\
\hline \multirow{5}{*}{30,0} & 0,00 & 0,00 & 265,00 & $23,19 \mathrm{O}$ & 5,24 & $26,95 \mathrm{~L}$ & 7,65 \\
\hline & 1,00 & 0,00 & 272,00 & $26,74 \mathrm{LM}$ & 7,81 & $34,68 \mathrm{GH}$ & 6,17 \\
\hline & 2,00 & 0,00 & 270,00 & $25,73 \mathrm{M}$ & 2,83 & $35,73 \mathrm{FG}$ & 2,03 \\
\hline & 3,00 & 0,00 & 275,00 & $27,23 \mathrm{LM}$ & 5,18 & $33,46 \mathrm{GHI}$ & 3,98 \\
\hline & 4,50 & 0,00 & 275,00 & $23,47 \mathrm{O}$ & 5,63 & $31,56 \mathrm{IJ}$ & 6,39 \\
\hline \multirow{5}{*}{40,0} & 0,00 & 0,00 & 264,00 & $16,95 \mathrm{R}$ & 4,57 & $26,83 \mathrm{~L}$ & 6,44 \\
\hline & 1,00 & 0,00 & 262,00 & $20,02 \mathrm{Q}$ & 5,95 & $28,37 \mathrm{KL}$ & 5,38 \\
\hline & 2,00 & 0,00 & 270,00 & $23,61 \mathrm{O}$ & 5,49 & $34,58 \mathrm{GH}$ & 3,00 \\
\hline & 3,00 & 0,00 & 260,00 & $22,47 \mathrm{OP}$ & 6,65 & $31,87 \mathrm{IJ}$ & 5,40 \\
\hline & 4,50 & 0,00 & 253,00 & $21,16 \mathrm{PQ}$ & 5,04 & $30,55 \mathrm{JK}$ & 1,32 \\
\hline
\end{tabular}

Letras diferentes denotam diferenças estatísticas entre as médias nas colunas ao nível de 95\% de confiança; TS-CV: Teor de substituição da cinza volante; T-CC: Teor de $\mathrm{CaCl}_{2} \cdot 2 \mathrm{H}_{2} \mathrm{O}$; T-SP: Teor de superplastificante; CONS: Consistência; CVar: coeficiente de variação; $\mathrm{PN}^{1}$ Ref.1: Pinus spp. in natura; PNCC ${ }^{2}$ Ref.2: Pinus spp. in natura $+4,5 \% \mathrm{CaCl}_{2} \cdot 2 \mathrm{H}_{2} \mathrm{O} ; \mathrm{PNAFCC}^{3} \mathrm{Ref} 3$ : Pinus spp. in natura com 48 horas de tratamento em água fria $+4,5 \% \mathrm{CaCl}_{2} \cdot 2 \mathrm{H}_{2} \mathrm{O}$. 
A superfície de resposta apresentada na figura 1 demonstra que a RC 91d do compósito decresce acentuadamente para teores de cinza volante (CV) acima de 5\%, corroborando o verificado nas determinações da resistência à compressão aos 91 dias de cura apresentadas na tabela 1 . Verifica-se, também, que a RC 91d independe do teor de $\mathrm{CaCl}_{2} \cdot 2 \mathrm{H}_{2} \mathrm{O}$, o que é confirmado através da análise numérica da equação ${ }^{\circ} 2$.

Procedendo à otimização da função, dentro dos limites impostos pelas condições de produção do compósito, chega-se à seguinte composição: cimento $=95 \%$, cinza volante $=5 \%$ e $\mathrm{CaCl}_{2} \cdot 2 \mathrm{H}_{2} \mathrm{O}=0 \%$. A expectativa de resistência à compressão para essa composição otimizada, aos 91 dias de idade, determinada pelo modelo, foi de 48,5027 MPa.

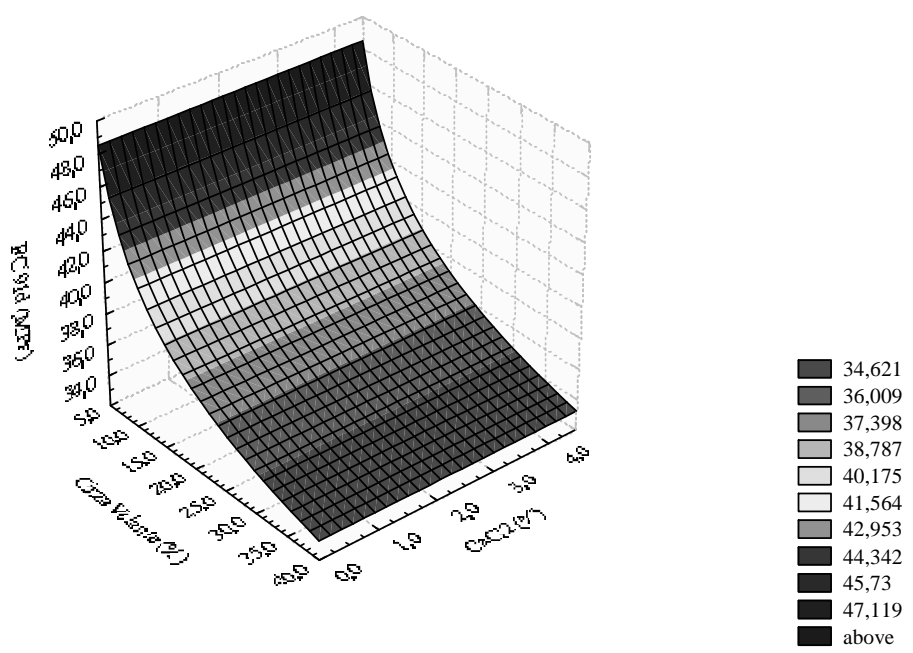

Figura 1. Variação da resistência à compressão aos 91 dias de idade em função da substituição parcial do cimento Portland por cinza volante e do teor de $\mathrm{CaCl}_{2} \cdot 2 \mathrm{H}_{2} \mathrm{O}$.

Figure 1. Variation of compression strength to age of 91 days in function of partial substitution of Portland cement by fly ash and amount of $\mathrm{CaCl}_{2} \cdot 2 \mathrm{H}_{2} \mathrm{O}$.

\section{Análise da substituição parcial do cimento Portland por escória de alto-forno (EAF)}

Na tabela 2 estão apresentados os valores das RC 7d e RC 91d para as substituições parciais do cimento Portland por teores de $25 \%, 30 \%, 35 \%, 40 \%, 50 \%$ e $60 \%$ de EAF.

A análise dos resultados apresentados indica que os maiores valores de $\mathrm{RC} 7 \mathrm{~d}$, para os compósitos produzidos com substituição parcial do cimento Portland por EAF, se verificaram para os compósitos com EAF $25 \%$ com adição de $3 \%$ e $4,5 \%$ de $\mathrm{CaCl}_{2} \cdot 2 \mathrm{H}_{2} \mathrm{O}$ e EAF $30 \%$ com adição de $3 \%$ de $\mathrm{CaCl}_{2} \cdot 2 \mathrm{H}_{2} \mathrm{O}$, sendo que tais valores não apresentaram diferenças estatísticas entre si, mas são significativamente superiores aos determinados com os três compósitos de Referência.

Para os ensaios com 91 dias de idade, as maiores resistências à compressão foram verificadas para os compósitos EAF $25 \%$, com adição de $3 \%$ de $\mathrm{CaCl}_{2} \cdot 2 \mathrm{H}_{2} \mathrm{O}$, EAF $35 \%$ com $2 \%$ e $3 \%$ de $\mathrm{CaCl}_{2} \cdot 2 \mathrm{H}_{2} \mathrm{O}$ e EAF $40 \%$ com $2 \%$ e $3 \%$ de $\mathrm{CaCl}_{2} \cdot 2 \mathrm{H}_{2} \mathrm{O}$, sendo que todas elas foram significativamente superiores em relação aos três compósitos de Referência. Os resultados indicam que é possível substituir até 50\% do cimento Portland do compósito por EAF sem que haja diferenças estatísticas significativas dos valores determinados para a maior resistência determinada com os compósitos de Referência aos 91 dias de idade (Ref. 3).

O modelo que expressa a dependência da resistência à compressão do compósito aos 91 dias, em função dos teores de substituição do cimento por EAF, de $\mathrm{CaCl}_{2} \cdot 2 \mathrm{H}_{2} \mathrm{O}$ e de aditivo superplastificante, é apresentado na equação 3 .

$$
\operatorname{RC} 91 \mathrm{~d}(\mathrm{MPa})=1 /\left(1,9954 \times 10^{-2}+4,0104 \times 10^{-8} \times[\operatorname{Teor} \operatorname{EAF}(\%)]^{3}+2,8593 \times 10^{-3} \times \operatorname{Exp}(-[\operatorname{Teor} \operatorname{CaCl}(\%)])\right)
$$

O modelo apresentou forte correlação $\left(R=0,8721, R^{2}=0,7605\right.$ e $\left.R^{2}{ }_{\text {Ajust }}=0,7564\right)$, o que indica que os dados experimentais estão, também, bem ajustados pela superfície de resposta. A confiabilidade do modelo, calculada com base na sua significância $\left(4,8 \times 10^{-35}\right)$, é superior a 99,99\%. 
Tabela 2. Variação da resistência à compressão aos 7 e 91 dias de idade em função da substituição parcial do cimento Portland por EAF e do teor de $\mathrm{CaCl}_{2} \cdot 2 \mathrm{H}_{2} \mathrm{O}$.

Table 2. Variation of compression strength to ages of 7 and 91 days in function of partial substitution of Portland cement by blast slag furnace and amount of $\mathrm{CaCl}_{2} \cdot 2 \mathrm{H}_{2} \mathrm{O}$.

\begin{tabular}{|c|c|c|c|c|c|c|c|}
\hline \multirow{2}{*}{ TS-EAF } & \multirow{2}{*}{$\begin{array}{l}\text { T-CC } \\
(\%)\end{array}$} & \multirow{2}{*}{$\begin{array}{l}\text { T-SP } \\
(\%)\end{array}$} & \multirow{2}{*}{$\begin{array}{l}\text { CONS } \\
(\mathrm{mm})\end{array}$} & \multicolumn{4}{|c|}{ Resistência à compressão (MPa) } \\
\hline & & & & RC-7d & CVar $(\%)$ & RC-91d & CVar (\%) \\
\hline $\mathrm{PN}^{2}$ & 0,00 & 0,00 & 283,00 & $20,01 \mathrm{Q}$ & 4,99 & $28,97 \mathrm{~S}$ & 5,68 \\
\hline $\mathrm{PNCC}^{3}$ & 4,50 & 0,00 & 257,00 & $32,57 \mathrm{FGH}$ & 1,90 & $39,82 \mathrm{LMN}$ & 5,46 \\
\hline PNAFCC $^{4}$ & 4,50 & 0,00 & 248,00 & $34,73 \mathrm{ABCD}$ & 2,48 & $43,91 \mathrm{GH}$ & 1,21 \\
\hline \multirow{5}{*}{25,0} & 0,00 & 0,00 & 270,00 & $32,21 \mathrm{IJK}$ & 3,24 & 42,09HIJKL & 2,88 \\
\hline & 1,00 & 0,00 & 270,00 & $37,85 \mathrm{C}$ & 2,81 & $45,32 \mathrm{EFG}$ & 2,82 \\
\hline & 2,00 & 0,00 & 270,00 & $38,01 \mathrm{BC}$ & 4,79 & $44,28 \mathrm{FGH}$ & 1,92 \\
\hline & 3,00 & 0,00 & 270,00 & $40,88 \mathrm{~A}$ & 3,84 & $48,83 \mathrm{AB}$ & 1,92 \\
\hline & 4,50 & 0,00 & 270,00 & $39,78 \mathrm{AB}$ & 4,17 & $46,87 \mathrm{BCDE}$ & 2,82 \\
\hline \multirow{5}{*}{30,0} & 0,00 & 0,00 & 275,00 & $27,61 \mathrm{MN}$ & 6,00 & $39,46 \mathrm{MN}$ & 5,92 \\
\hline & 1,00 & 0,00 & 270,00 & 33,79 FGHIJ & 6,06 & 40,58 JKLMN & 2,56 \\
\hline & 2,00 & 0,00 & 270,00 & $38,19 \mathrm{BC}$ & 3,13 & 45,24 EFG & 3,54 \\
\hline & 3,00 & 0,00 & 270,00 & $36,87 \mathrm{CD}$ & 3,53 & 45,54 DEFG & 3,51 \\
\hline & 4,50 & 0,00 & 267,00 & $40,52 \mathrm{~A}$ & 2,66 & 42,70 HIJ & 5,62 \\
\hline \multirow{5}{*}{35,0} & 0,00 & 0,00 & 270,00 & $26,42 \mathrm{NO}$ & 5,28 & 41,84 IJKLM & 2,29 \\
\hline & 1,00 & 0,00 & 267,00 & 29,21 LM & 6,43 & 46,40 CDEF & 1,12 \\
\hline & 2,00 & 0,00 & 266,50 & $34,27 \mathrm{EFGH}$ & 5,72 & $47,76 \mathrm{ABCD}$ & 1,90 \\
\hline & 3,00 & 0,00 & 266,00 & 35,33 DEF & 7,30 & $49,52 \mathrm{~A}$ & 1,44 \\
\hline & 4,50 & 0,00 & 265,00 & $35,86 \mathrm{DE}$ & 3,55 & 46,67 BCDEF & 3,08 \\
\hline \multirow{5}{*}{40,0} & 0,00 & 0,00 & 274,00 & $24,75 \mathrm{OP}$ & 5,51 & 42,28 HIJK & 4,03 \\
\hline & 1,00 & 0,00 & 273,00 & 33,26 GHIJ & 2,90 & $45,12 \mathrm{DEFG}$ & 1,72 \\
\hline & 2,00 & 0,00 & 269,00 & 34,44 EFGH & 1,58 & $48,07 \mathrm{ABC}$ & 3,10 \\
\hline & 3,00 & 0,00 & 276,00 & 34,06 EFGHI & 3,54 & 47,14 ABCDE & 3,06 \\
\hline & 4,50 & 0,00 & 276,00 & $35,17 \mathrm{DEF}$ & 5,09 & 46,19 CDEFG & 2,02 \\
\hline \multirow{5}{*}{50,0} & 0,00 & 0,00 & 260,00 & $21,48 \mathrm{Q}$ & 5,15 & $36,12 \mathrm{OP}$ & 6,78 \\
\hline & 1,00 & 0,00 & 265,00 & $23,62 \mathrm{P}$ & 3,92 & 40,27 KLMN & 5,40 \\
\hline & 2,00 & 0,00 & 265,00 & $28,02 \mathrm{MN}$ & 2,69 & 41,97 HIJKL & 5,47 \\
\hline & 3,00 & 0,00 & 270,00 & $32,00 \mathrm{JK}$ & 2,58 & 40,57 JKLMN & 7,70 \\
\hline & 4,50 & 0,00 & 268,00 & 33,01 GHIJ & 3,68 & $38,53 \mathrm{NO}$ & 4,83 \\
\hline \multirow{5}{*}{60,0} & 0,00 & 0,00 & 282,00 & $20,59 \mathrm{Q}$ & 2,61 & $31,02 \mathrm{RS}$ & 5,59 \\
\hline & 1,00 & 0,00 & 280,00 & $23,41 \mathrm{P}$ & 2,77 & $33,19 \mathrm{QR}$ & 4,97 \\
\hline & 2,00 & 0,00 & 282,00 & $27,60 \mathrm{MN}$ & 3,87 & $35,07 \mathrm{PQ}$ & 3,05 \\
\hline & 3,00 & 0,00 & 280,00 & 29,13 LM & 5,56 & $34,86 \mathrm{PQ}$ & 7,39 \\
\hline & 4,50 & 0,00 & 278,00 & $30,82 \mathrm{KL}$ & 3,47 & $32,79 \mathrm{QR}$ & 4,99 \\
\hline
\end{tabular}

Letras diferentes denotam diferenças estatísticas entre as médias nas colunas ao nível de 95\% de confiança; TS-EAF: Teor de substituição da escória de alto-forno; T-CC: Teor de $\mathrm{CaCl}_{2} .2 \mathrm{H}_{2} \mathrm{O}$; T-SP: Teor de superplastificante; CONS: Consistência; CVar: coeficiente de variação; $\mathrm{PN}^{1}$ Ref.1: Pinus spp. in natura; $\mathrm{PNCC}^{2}$ Ref.2: Pinus spp. in natura $+4,5 \% \mathrm{CaCl}_{2} .2 \mathrm{H}_{2} \mathrm{O}$; $\mathrm{PNAFCC}^{3}$ Ref.3: Pinus spp. in natura com 48 horas de tratamento em água fria $+4,5 \% \mathrm{CaCl}_{2} \cdot 2 \mathrm{H}_{2} \mathrm{O}$.

Na figura 2 está apresentada a superfície de resposta da RC 91d do compósito com substituição parcial do cimento Portland CPV ARI RS, em função do teor de substituição por EAF e do teor de $\mathrm{CaCl}_{2} \cdot 2 \mathrm{H}_{2} \mathrm{O}$, conforme modelo determinado pela análise multivariada.

A superfície de resposta demonstra que há uma variação da ordem de 59,64\% na RC 91d dos compósitos produzidos com substituições do cimento Portland por teores de EAF, variando de 49,52 MPa (35\% de EAF e 3,0\% de $\mathrm{CaCl}_{2} \cdot 2 \mathrm{H}_{2} \mathrm{O}$ ) a $31,02 \mathrm{MPa}\left(60 \%\right.$ de EAF e 0,0\% de $\mathrm{CaCl}_{2} \cdot 2 \mathrm{H}_{2} \mathrm{O}$ ).

Procedendo à otimização da função, dentro dos limites impostos pelas condiçães de produção do compósito, chega-se à seguinte composição: cimento $=75 \%, \mathrm{EAF}=25 \%, \mathrm{CaCl}_{2} \cdot 2 \mathrm{H}_{2} \mathrm{O}=4,5 \% \mathrm{e}$ superplastificante $=0,0 \%$. A expectativa de resistência à compressão para essa composição otimizada, aos 91 dias de idade, determinada pelo modelo, é de 48,51MPa. 


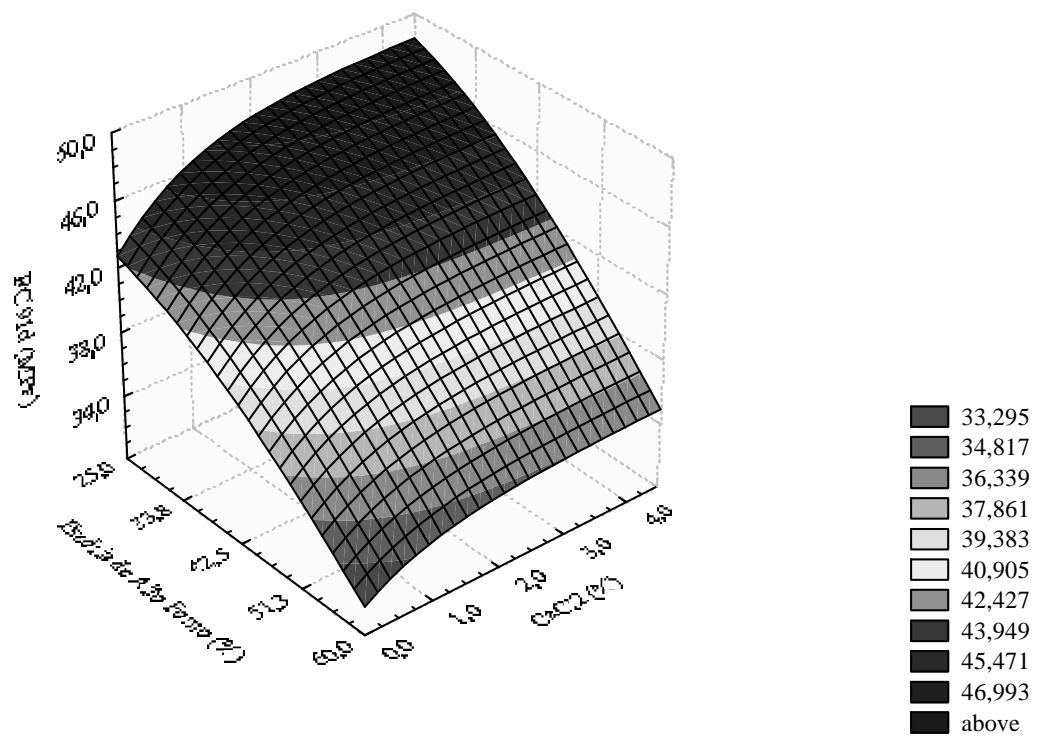

Figura 2. Variação da resistência à compressão aos 91 dias de idade em função da substituição parcial do cimento Portland por EAF e do teor de $\mathrm{CaCl}_{2} \cdot 2 \mathrm{H}_{2} \mathrm{O}$.

Figure 2. Variation of compression strength to age of 91 days in function of partial substitution of Portland cement by EAF and amount of $\mathrm{CaCl}_{2} \cdot 2 \mathrm{H}_{2} \mathrm{O}$.

\section{Análise dos compósitos com substituições parciais do cimento Portland otimizadas}

Para comprovar as expectativas de RC 91d e de determinar as demais características físicas e mecânicas dos compósitos cimento-madeira produzidos com o resíduo de Pinus spp. e com as substituições parciais do cimento Portland, procedeu-se a produção e teste dos compósitos.

$\mathrm{Na}$ tabela 3 estão apresentadas as características físicas dos compósitos produzidos com as substituições parciais do cimento Portland e dos compósitos de Referência.

Com relação às características físicas dos compósitos, contatou-se que a substituição parcial do cimento Portland pela CV e pela EAF não contribuiu para alterações importantes das características físicas dos compósitos, como pode ser evidenciado nos gráficos apresentados na figura 3.

Tabela 3. Compósitos com resíduo de Pinus spp. e substituições parciais do cimento Portland Características físicas.

Table 3. Composites with residues of Pinus spp. and partial substitution of Portland cement - Physical characteristics.

\begin{tabular}{lcccccc}
\hline Compósito & $\begin{array}{c}\text { ME } \\
\left(\mathbf{k g f} / \mathbf{m}^{3}\right)\end{array}$ & $\begin{array}{c}\text { CVar } \\
(\boldsymbol{\%})\end{array}$ & $\begin{array}{c}\text { IV } \\
(\boldsymbol{\%})\end{array}$ & $\begin{array}{c}\text { CVar } \\
(\boldsymbol{\%})\end{array}$ & $\begin{array}{c}\text { ABS } \\
(\boldsymbol{\%})\end{array}$ & $\begin{array}{c}\text { CVar } \\
(\boldsymbol{\%})\end{array}$ \\
\hline $\mathrm{PN}^{1}$ & $1.471,10 \mathrm{C}$ & 0,45 & $38,98 \mathrm{~A}$ & 0,74 & $26,49 \mathrm{~A}$ & 0,79 \\
PNCC $^{2}$ & $1.594,17 \mathrm{~A}$ & 0,62 & $36,29 \mathrm{C}$ & 1,15 & $22,77 \mathrm{C}$ & 3,04 \\
PNAFCC $^{3}$ & $1.503,79 \mathrm{~B}$ & 0,88 & $35,77 \mathrm{CD}$ & 0,81 & $23,79 \mathrm{~B}$ & 1,61 \\
CV 5\% & $1.474,26 \mathrm{C}$ & 0,75 & $37,78 \mathrm{~B}$ & 2,92 & $25,63 \mathrm{~A}$ & 3,48 \\
EAF 25\% & $1.503,79 \mathrm{~B}$ & 0,27 & $34,71 \mathrm{D}$ & 2,04 & $23,09 \mathrm{BC}$ & 2,57 \\
\hline
\end{tabular}

Letras diferentes denotam diferenças estatísticas entre as médias nas colunas ao nível de 95\% de confiança. ME: massa específica; CVar: coeficiente de variação; IV: índice de vazios; ABS: absorção; $\mathrm{PN}^{1}$ Ref.1: Pinus spp. in natura; PNCC ${ }^{2}$ Ref.2: Pinus spp. in natura $+4,5 \% \mathrm{CaCl}_{2} \cdot 2 \mathrm{H}_{2} \mathrm{O}$; PNAFCC ${ }^{3}$ Ref.3: Pinus spp. in natura com 48 horas de tratamento em água fria $+4,5 \% \mathrm{CaCl}_{2} \cdot 2 \mathrm{H}_{2} \mathrm{O}$; CV: Cinza volante; EAF: Escória de alto-forno.

Na figura 3 estão apresentados os gráficos relativos às características físicas dos compósitos de referência e dos otimizados, em relação à substituição parcial do cimento Portland pelos seis materiais cimentícios alternativos. 

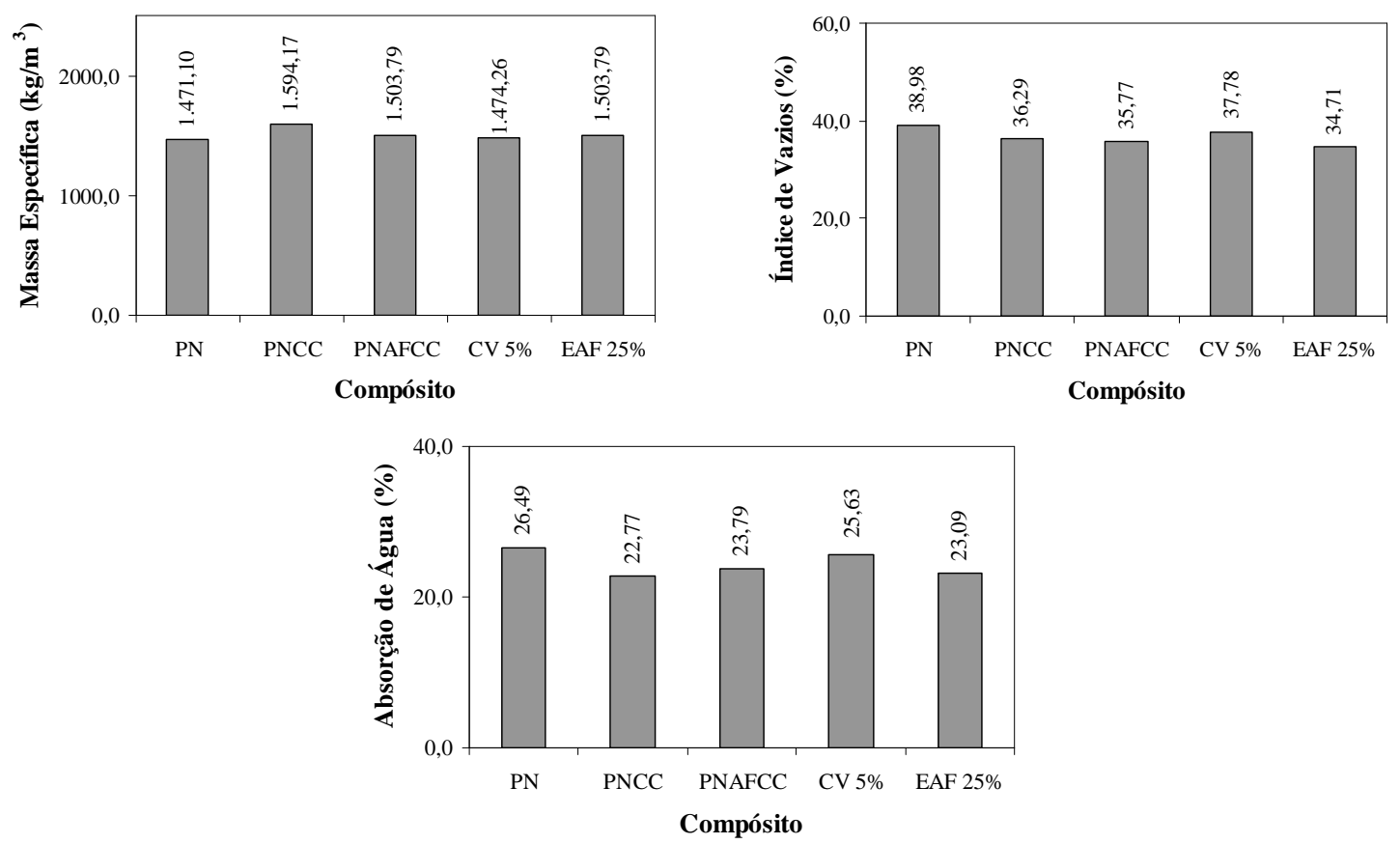

Figura 3. Características físicas dos compósitos com resíduo de Pinus spp. e substituições parciais do cimento Portland por CV e EAF.

Figure 3. Physical characteristics of composites with residue of Pinus spp. and partial substitutions of Portland cement by $\mathrm{CV}$ and EAF.

$\mathrm{Na}$ tabela 4 são apresentadas as características mecânicas dos compósitos produzidos com substituições parciais do cimento por CV e EAF.

Tabela 4. Compósitos com resíduo de Pinus spp. e substituições parciais do cimento Portland por CV e EAF - Características mecânicas.

Table 4. Composites with residues of Pinus spp. partial substitution of Portland cement by CV and EAF - Mechanical characteristics.

\begin{tabular}{lcccccccc}
\hline \multirow{2}{*}{ Compósito } & \multicolumn{4}{c}{ Resistência à compressão - RC } & \multicolumn{3}{c}{ Resistência à tração } \\
\cline { 2 - 10 } & $\begin{array}{c}\text { RC 7d } \\
(\mathbf{M P a})\end{array}$ & $\begin{array}{c}\text { CVar } \\
(\boldsymbol{\%})\end{array}$ & $\begin{array}{c}\text { RC 28d } \\
(\mathbf{M P a})\end{array}$ & $\begin{array}{c}\text { CVar } \\
(\boldsymbol{\%})\end{array}$ & $\begin{array}{c}\text { RC 91d } \\
(\mathbf{M P a})\end{array}$ & $\begin{array}{c}\text { CVar } \\
(\boldsymbol{\%})\end{array}$ & $\begin{array}{c}\text { RT 28d } \\
(\mathbf{M P a})\end{array}$ & $\begin{array}{c}\text { CVar } \\
(\%)\end{array}$ \\
\hline PN $^{1}$ & $20,01 \mathrm{D}$ & 4,99 & $24,59 \mathrm{D}$ & 1,29 & $28,97 \mathrm{D}$ & 5,68 & $1,95 \mathrm{E}$ & 2,33 \\
PNCC $^{2}$ & $32,57 \mathrm{~B}$ & 1,90 & $38,64 \mathrm{~B}$ & 3,60 & $39,82 \mathrm{C}$ & 5,46 & $2,57 \mathrm{D}$ & 2,63 \\
PNAFCC $^{3}$ & $34,73 \mathrm{~A}$ & 2,48 & $42,72 \mathrm{~A}$ & 2,69 & $43,91 \mathrm{~B}$ & 1,21 & $2,67 \mathrm{D}$ & 2,13 \\
CV 5\% & $29,72 \mathrm{C}$ & 4,94 & $37,96 \mathrm{~B}$ & 2,59 & $47,52 \mathrm{~A}$ & 3,29 & $3,84 \mathrm{~A}$ & 4,08 \\
EAF 25\% & $32,18 \mathrm{~B}$ & 4,40 & $38,29 \mathrm{~B}$ & 4,95 & $43,03 \mathrm{~B}$ & 3,04 & $3,65 \mathrm{~B}$ & 5,38 \\
\hline
\end{tabular}

Letras diferentes denotam diferenças estatísticas entre as médias nas colunas ao nível de $95 \%$ de confiança; CVar: coeficiente de variação; $\mathrm{PN}^{1}$ Ref.1: Pinus spp. in natura; $\mathrm{PNCC}^{2} \mathrm{Ref} .2$ : Pinus spp. in natura $+4,5 \% \mathrm{CaCl}_{2} \cdot 2 \mathrm{H}_{2} \mathrm{O}$; PNAFCC ${ }^{3}$ Ref.3: Pinus spp. in natura com 48 horas de tratamento em água fria $+4,5 \% \mathrm{CaCl}_{2} \cdot 2 \mathrm{H}_{2} \mathrm{O}$; $\mathrm{SA}$ : sílica ativa; $\mathrm{CCA}$ : cinza de casca de arroz.

Conforme os resultados determinados quanto às resistências à compressão, pode-se constatar:

- Os maiores valores de resistência à compressão aos 7 e 28 dias de idade foram obtidos para o compósito Ref 3. Para 91 dias de idade, foram verificadas algumas alterações nos resultados de resistência à compressão dos compósitos. O compósito produzido com $5 \%$ de $\mathrm{CV}$ passou a apresentar maior RC, e o compósito com $25 \%$ de EAF passou a não apresentar diferença estatística significativa em relação à RC do compósito Ref 3 , comprovando os efeitos de maior tempo para combinação do $\mathrm{Ca}(\mathrm{OH})_{2}$ do cimento com os materiais cimentícios alternativos. 
- Os menores valores de resistência à compressão, para todas as idades, foram obtidos para o compósito produzido com $100 \%$ de cimento Portland e com o resíduo de Pinus spp. in natura.

- A evolução das resistências à compressão dos compósitos com substituição parcial do cimento Portland CPV ARI RS pelos teores otimizados de CV (5\%) e EAF (25\%), em comparação aos valores obtidos para os compósitos de Referência, podem ser verificadas na figura 4.

- Quanto aos resultados de resistência à tração apresentados na tabela 4 , pode-se verificar que houve diferenças significativas entre os valores das resistências à tração determinados para os compósitos.

- O compósito produzido com substituição parcial do cimento Portland por 5\% de CV apresentou a maior RT 28d (3,84 MPa), sendo este valor 96,92\% maior que a Ref 1, 49,46\% maior que a Ref 2 e $43,82 \%$ maior que a Ref 3 .

- Quanto à substituição parcial por 25\% de EAF, a RT 28d (3,65 MPa) determinada foi 87,18\% maior que a determinada para a Ref $1,42,02 \%$ maior que a Ref 2 e 36,70\% maior que os valores determinados para a Ref 3 .

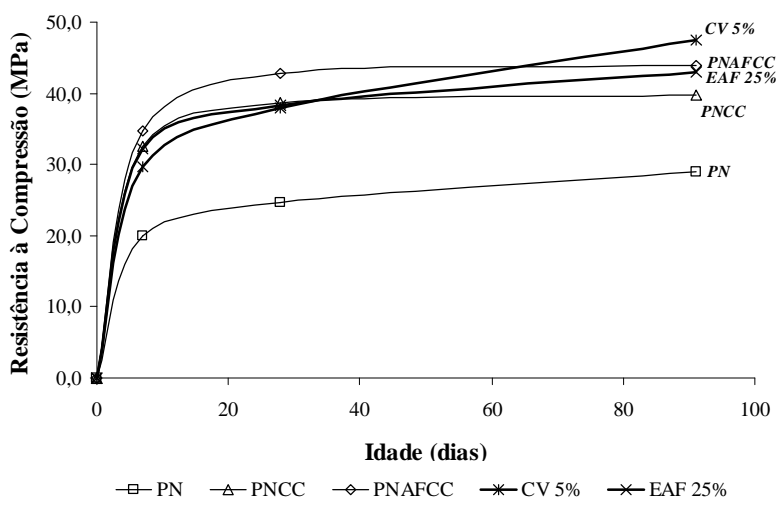

Figura 4. Compósitos com resíduo de Pinus spp. e substituições parciais do cimento Portland - Evolução da resistência à compressão com a idade.

Figure 4. Composites with residues of Pinus spp. and partial substitutions of Portland cement Evolution of compression strength by ages.

Na figura 5, apresenta-se a comparação entre os valores determinados para a resistência à tração, determinada por compressão diametral dos corpos de prova, na idade de 28 dias.

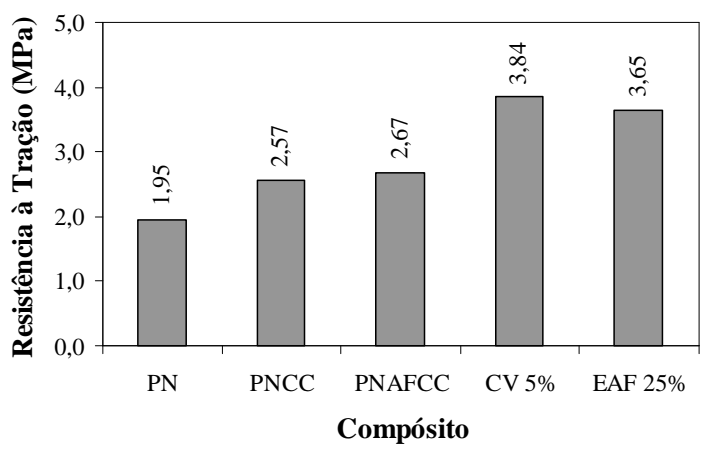

Figura 5. Compósitos com resíduo de Pinus spp. e substituições parciais do cimento Portland Resistência à tração.

Figure 5. Composites with residues of Pinus spp. and partial substitutions of Portland cement - Tension strength. 


\section{CONCLUSÕES}

Com base nos resultados obtidos nesta pesquisa, as seguintes conclusões podem ser apresentadas:

- Diante das características físicas e mecânicas dos compósitos produzidos, conclui-se que a utilização do resíduo de Pinus spp. com granulometria semelhante à do agregado miúdo mineral convencional (areia com Dmáx $=4,8 \mathrm{~mm}$ ) é tecnicamente viável para a produção de compósitos cimento-madeira.

- A utilização de $\mathrm{CaCl}_{2} \cdot 2 \mathrm{H}_{2} \mathrm{O}$ como aditivo acelerador de pega propiciou ganhos significativos de resistência dos compósitos de Referência, produzidos com $100 \%$ de cimento Portland.

- A utilização de pré-tratamento, do tipo extração em água fria por 48 horas (AF-48H), propiciou aumentos significativos da resistência à compressão dos compósitos quando comparados aos compósitos com resíduo de Pinus spp. sem tratamento.

- O teor ótimo de substituição do cimento Portland por cinza volante, determinado através de análise multivariada, foi de $5 \%$, com $0 \%$ de $\mathrm{CaCl}_{2} \cdot 2 \mathrm{H}_{2} \mathrm{O}$, o que indica que, nos compósitos produzidos com substituições parciais do cimento pela cinza volante (CV), o aditivo acelerador de pega utilizado não apresenta influência sobre as características mecânicas.

- O teor ótimo de substituição do cimento Portland por escória de alto-forno (EAF), determinado através de análise multivariada, foi de $25 \%$ de $\mathrm{EAF}$ e $4,5 \%$ de $\mathrm{CaCl}_{2} \cdot 2 \mathrm{H}_{2} \mathrm{O}$, o que denota a influência diretamente proporcional do teor de aditivo acelerador de pega utilizado sobre as características mecânicas dos compósitos produzidos.

- Considerando as RC 91d médias determinadas com os compósitos de Referência, verifica-se a viabilidade de substituição do cimento Portland dos compósitos por até $10 \%$ de CV e 50\% de EAF.

- As características físicas dos compósitos produzidos com substituições parciais de CV e EAF pouco diferiram das características dos compósitos de Referência.

- O compósito produzido com a substituição parcial otimizada do cimento Portland por CV apresentou RC 91d e RT 28d superiores aos compósitos de Referência, produzidos com 100\% de cimento Portland.

- O compósito produzido com a substituição parcial otimizada do cimento Portland por EAF apresentou RC 91d sem diferenças estatísticas significativas às determinadas pelo compósito Ref. 3, e RT 28d significativamente superior às resistências à tração determinadas para aos três traços de Referência, produzidos com $100 \%$ de cimento Portland.

- Os resultados da pesquisa comprovaram a possibilidade de uso de cinza volante e escória de alto-forno em substituições parciais do cimento Portland, em proporções de $5 \%$ e de $25 \%$, respectivamente, na produção de compósitos cimento-madeira. A viabilidade de uso de resíduos minerais em substituição ao cimento Portland contribui de forma significativa em termos de impacto ambiental.

\section{REFERÊNCIAS}

AÏTCIN, P. C. Concreto de alto desempenho. São Paulo: Editora Pini, 2000. 667 p.

ASSOCIAÇÃO BRASILEIRA DA INDÚSTRIA DE MADEIRA PROCESSADA MECANICAMENTE (ABIMCI). Estudo Setorial 2008. Disponível em: <http://www.abimci.com.br>. Acesso em 25 set. 2009.

ASSOCIAÇÃO BRASILEIRA DE NORMAS TÉCNICAS (ABNT). NBR 5733: Cimento Portland de alta resistência inicial. Rio de Janeiro, 1991.

. NBR 5735: Cimento Portland de alto-forno. Rio de Janeiro, 1991.

. NBR 5736: Cimento Portland pozolânico. Rio de Janeiro, 1991.

NBR 7215: Cimento Portland - Determinação da resistência à compressão. Rio de Janeiro, 1991.

NBR 7222: Argamassa e concreto - Determinação da resistência à tração por compressão diametral de corpos de prova cilíndricos. Rio de Janeiro, 1991. 1991.

. Argamassa e concreto - Câmaras úmidas e tanques para cura de corpos de prova. Rio de Janeiro, 
NBR 9778: Argamassas e concretos endurecidos - Determinação da absorção de água, índice de vazios e massa específica. Rio de Janeiro, 1991.

NBR 11578: Cimento Portland composto. Rio de Janeiro, 1991.

NBR 12653: Materiais pozolânicos. Rio de Janeiro, 1991.

CARMO, J. B. M. Análise comparativa do desempenho de concretos compostos com sílica ativa e metacaulim face à resistência mecânica e à viabilidade econômica. Curitiba, 2006. 136 p. Dissertação (Mestrado) - Universidade Federal do Paraná.

FREITAS, L. C. A baixa produtividade e o desperdício no processo de beneficiamento da madeira: um estudo de caso. 2000. 120 f. Dissertação (Mestrado em Engenharia Civil) - Universidade Federal de Santa Catarina, Florianópolis, 2000.

FREITAS JÚNIOR, J. A. Estudo comparativo de métodos de dosagem para concretos de alta resistência com o uso de materiais disponíveis na região metropolitana de Curitiba. $2005.131 \mathrm{f}$. Dissertação (Mestrado em Construção Civil) - Universidade Federal do Paraná, Curitiba, 2005.

ISAIA, G. C. Concreto: ensino, pesquisa e realizações. São Paulo: IBRACON, 2005. 1.600 p.

IWAKIRI, S. Painéis de madeira reconstituída. Curitiba: Fundação de Pesquisas Florestais do Paraná FUPEF, 2005, $247 \mathrm{p}$.

JOHN, V. M.; AGOPYAN, V. Materiais reforçados com fibras vegetais. In: SIMPÓSIO INTERNACIONAL DE MATERIAIS REFORÇADOS COM FIBRAS PARA A CONSTRUÇÃO, São Paulo, 1993. Anais... São Paulo: EPUSP, 1993. p. 29 - 38.

LANGE, H.; SIMATUPANG, M. H.; NEUBAUER, A. Influence of latent hydraulic binders on the properties of wood-cement composites. In: $1^{\text {st }}$ Inorganic Bonded Fiber Composites Symposium. Forest Products Research Society. Proceedings... p. 48 - 52, USA. 1989.

LIMA, A. F.; JARÁ, E. R. P.; ALFONSO, V. A. Madeira como matéria-prima para fabricação de pasta celulósica. In: Celulose e Papel: tecnologia de fabricação da pasta celulósica, 2, 1988, São Paulo. São Paulo: IPT, 1988, p. 129 - 167.

MEHTA, P. K.; MONTEIRO, P. J. M. Concreto: estrutura, propriedades e materiais. São Paulo: IBRACON, 2008, 674 p.

MORAIS, S. A. L.; NASCIMENTO, E. A.; MELO, D. C. Análise da madeira de Pinus oocarpa Parte I Estudo dos constituintes macromoleculares e extrativos voláteis. Revista Árvore, Viçosa, v. 29, n. 3, p. $461-470,2005$.

MOSLEMI. A. A. Wood-cement panel products: coming of age. In: 1st Inorganic Bonded Fiber Composites Symposium. Forest Products Research Society. USA. Proceedings... 1989, USA: 1989, p. 12 - 18.

NAHUZ, M. A. R. Atividades industriais com madeiras de Pinus - Atualidades e desafios. Revista da Madeira, Curitiba, n. 83, p. 10 - 12, 2004.

OLIVEIRA, C. T. A. Água do poro de pastas de cimento de escória. 2000. 162 p. Tese (Doutorado em Engenharia Civil) - Escola Politécnica da Universidade de São Paulo, São Paulo, 2000.

RAMIREZ-CORETTI, A.; ECKELMAN, C. A.; WOLF, R. W. Inorganic-bonded composite wood panel systems for low-cost housing: a Central American perspective. Forest Products Journal, Madison, n. 48, p. 62 - 68, 1998.

SANTOS, P. S. Ciência e tecnologia de argilas. 2. ed. São Paulo. Edgard Blücher, 1992.

SEMPLE, K. E.; EVANS, P. D. Wood-cement composites - Suitability of Western Australian mallee eucalypt, blue gum and melaleucas. Rural Industries Research and Development Corporation. Kingston: ACT, 2004. 64 p. 